\title{
Active Turbulence and Scalar Transport near the Forest-Atmosphere Interface
}

\author{
GABRIEL G. KATUL \\ School of the Environment and Center for Hydrologic Science, Duke University, Durham, North Carolina \\ Chris D. Geron \\ United States Environmental Protection Agency, Research Triangle Park, North Carolina \\ CHeng-I HSIEH \\ School of the Environment, Duke University, Durham, North Carolina \\ BRANI VIDAKOVIC \\ Institute of Statistics and Decision Sciences, Duke University, Durham, North Carolina \\ Alex B. Guenther \\ Atmospheric Chemistry Division, National Center for Atmospheric Research, Boulder, Colorado
}

(Manuscript received 25 August 1997, in final form 6 March 1998)

\section{ABSTRACT}

\begin{abstract}
Turbulent velocity, temperature, water vapor concentration, and other scalars were measured at the canopyatmosphere interface of a 13-14-m-tall uniform pine forest and a 33-m-tall nonuniform hardwood forest. These measurements were used to investigate whether the mixing layer (ML) analogy of Raupach et al. predicts eddy sizes and flow characteristics responsible for much of the turbulent stresses and vertical scalar fluxes. For this purpose, wavelet spectra and cospectra were derived and analyzed. It was found that the ML analogy predicts well vertical velocity variances and integral timescales. However, at low wavenumbers, inactive eddy motion signatures were present in horizontal velocity wavelet spectra, suggesting that ML may not be suitable for scaling horizontal velocity perturbations. Momentum and scalar wavelet cospectra of turbulent stresses and scalar fluxes demonstrated that active eddy motion, which was shown by Raupach et al. to be the main energy contributor to vertical velocity $(w)$ spectral energy $\left(E_{w}\right)$, is also the main scalar flux-transporting eddy motion. Predictions using ML of the peak $E_{w}$ frequency are in excellent agreement with measured wavelet cospectral peaks of vertical fluxes ( $K h=1.5$, where $K$ is wavenumber and $h$ is canopy height). Using Lorentz wavelet thresholding of vertical velocity time series, wavelet coefficients associated with active turbulence were identified. It was demonstrated that detection frequency of organized structures, as predicted from Lorentz wavelet filtering, relate to the arrival frequency $\langle U\rangle / h$ and integral timescale, where $\langle U\rangle$ is the mean horizontal velocity at height $z=h$. The newly proposed wavelet thresholding approach, which relies on a "global" wavelet threshold formulation for the energy in $w$, provides simultaneous energy-covariance-preserving characterization of "active" turbulence at the canopy-atmosphere interface.
\end{abstract}

\section{Introduction}

Understanding momentum, heat, and scalar mass exchanges between vegetation and the atmosphere is necessary for the quantification of evaporation and sensible heat flux for hydrologic budgets, ozone deposition on urban forests, nonmethane hydrocarbon emissions from

Corresponding author address: Gabriel G. Katul, School of the Environment, Box 902328, Duke University, Durham, NC 277080328.

E-mail: gaby@duke.edu natural vegetation, carbon storage in ecosystems, etc. Such exchanges are governed by a turbulent mixing process that appears to exhibit a number of universal characteristics (Amiro 1990; Amiro and Davis 1988; Raupach et al. 1989; Gao et al. 1992; Baldocchi and Meyers 1988, 1989; Paw U et al. 1992). Early attempts to predict these universal characteristics made use of rough-wall boundary layer analogies but limited success was reported (for review, see, e.g., Raupach et al. 1996; Raupach et al. 1991; Raupach 1979; Wilson 1989; Raupach and Thom 1981; Thompson 1979; Mulhearn and Finnigan 1978). A basic distinction between canopy and rough-wall boundary layer turbulence is that the "for- 
est-atmosphere" system is a porous medium permitting finite velocity and velocity perturbations well within the canopy. Hence, the canopy-atmosphere interface cannot impose a severe constraint on fluid continuity (the "no slip" boundary condition in specific) as an impervious boundary, as discussed in Raupach and Thom (1981), Raupach (1988), and Raupach et al. (1989).

Raupach et al. (1989) and Raupach et al. (1996) proposed a mixing layer (ML) analogy to model the universal characteristics of turbulence close to the canopy atmosphere interface in uniform and extensive canopies. Their analogy is based on solutions to the linearized perturbed two-dimensional inviscid momentum equations (Rayleigh's equation) using hydrodynamic stability theory (HST). For such a system of equations, HST predicts the unstable mode generation of two-dimensional transverse Kelvin-Helmholz $(\mathrm{KH})$ waves with streamwise wavelength $\left(\Lambda_{x}\right)$ if the longitudinal velocity profile has an inflection point (a necessary but not sufficient condition). Such instabilities are the origins of organized eddy motion in plane mixing layers; however, a KH eddy motion cannot be produced or sustained in boundary layers due to the absence of such an inflection point in the velocity profile. A plane mixing layer is a "boundary-free shear flow" formed in a region between two coflowing fluid streams of different velocity but same density (Tennekes and Lumley 1972, 104; Raupach et al. 1996). Raupach et al. (1996) recently argued that a strong inflection point in the mean velocity profile at the canopy-atmosphere interface results in a flow regime resembling a mixing layer rather than a boundary layer neighboring this interface. Raupach et al.'s (1996) ML analogy is the first theoretical advancement to analyzing the structure of turbulence close to the canopyatmosphere interface of a horizontally extensive uniform forest.

As discussed in Tennekes and Lumley (1972, 112), planar mixing layers are self-preserving so that mean velocity and turbulent stress profiles become invariant with respect to a horizontal distance $(x)$. Hence, such profiles can be expressed in terms of local length and velocity scales. The self-preservation hypothesis is consistent with numerous observations that turbulent mixing close to the canopy-atmosphere interface exhibits a number of universal characteristics. Among these universal characteristics are the emergence of friction velocity $\left(u_{*}\right)$ and canopy height $(h)$ as representative velocity and length scales (Raupach 1988; Raupach et al. 1989).

In addition to exerting drag on the airflow aloft, canopy foliage acts as a source (or sink) for many passive, active, and reactive scalar entities such as heat $\left(T_{a}\right)$, water vapor $(Q)$, ozone $\left(\mathrm{Q}_{3}\right)$, and isoprene (2-methyl1,3-butadiene, hereafter $I_{s}$ ) resulting in a "scalar mixing layer." A scalar mixing layer is a generalization of a thermal mixing layer, which is defined as a plane layer formed between two coflowing streams of same longitudinal velocity but different temperatures (LaRue and
Libby 1981; LaRue et al. 1981). Unfortunately, theoretical and laboratory investigations demonstrate that pure thermal mixing layers are not self-preserving (Lumley 1986). In fact, laboratory measurements of grid-generated turbulence in a thermal mixing layer (usually produced by heating one side of a grid) by LaRue and Libby (1981) and Ma and Warhaft (1986) illustrate that computations utilizing self-preservation assumptions overestimate the maximum measured heat flux by more than $25 \%$. Hence, instabilities responsible for momentum vertical transport may not transport scalars in an analogous manner.

Given the complexities of mean scalar concentration profiles within forested systems (see, e.g., Denmead and Bradley 1985; Moritz 1989), the dissimilarity in scalar and momentum sources and sinks, the large atmospheric stability variation close to the canopy-atmosphere interface, and the complex foliage distribution with height all suggest a need to further investigate the applicability of Raupach et al.'s (1996) ML analogy to scalar mass transport.

The objective of this study is to investigate whether the characteristics of active turbulence, typically identified from vertical velocity statistics, can be extended to mass transport at the canopy-atmosphere interface. In Raupach et al. (1996), particular attention was devoted to eddy sizes responsible for the generation of coherency and spectral peaks in the vertical velocity. Here, eddy sizes responsible for cospectral peaks of scalar fluxes and their relationship to active turbulence are considered. Active turbulence is identified from orthogonal wavelet decomposition that concentrates much of the vertical velocity energy in few wavelet coefficients. The remaining wavelet coefficients, associated with "inactive," "wake," and "finescale turbulence" are thresholded using a Lorentz wavelet approach advanced by Vidakovic (1995) and Katul and Vidakovic (1996, 1998). Wavelet spectra and cospectra are also used to investigate the characteristics of active turbulence for the two stands (different canopy heights, uniformity, and leaf area distribution) and for a wide range of atmospheric stability conditions. Much of the flow statistics derived by Raupach et al. (1996) in the time domain are also extended to the wavelet domain. Since canopy sublayer (CSL) turbulence is intermittent in the time domain with defined spectral properties in the Fourier domain, orthonormal wavelet decompositions permit a simultaneous time-frequency investigation of both flow characteristics.

Unless otherwise stated, the notation used throughout is as follows: $U_{i}\left(U_{1}=U, U_{2}=V\right.$, and $\left.U_{3}=W\right)$ are the instantaneous longitudinal $(U)$, lateral $(V)$, and vertical $(W)$ velocity components; $C$ is the instantaneous concentration of a scalar entity $\left(C=T_{a}, Q, \mathrm{CO}_{2}, \mathrm{O}_{3}\right.$, and $\left.\mathrm{I}_{\mathrm{s}}\right) ; x_{i}\left(x_{1}=x, x_{2}=y, x_{3}=z\right)$ are the longitudinal $(x)$, lateral $(y)$, and vertical $(z)$ directions with $z$ defined from the ground surface; $\langle\cdot\rangle$ is time averaging; $u_{i}$ and $c$ are the turbulent fluctuations around $\left\langle U_{i}\right\rangle$ and $\langle C\rangle$ so 
that $\left\langle u_{i}\right\rangle=\langle c\rangle=0$; and the $x$ direction is aligned along the mean horizontal wind direction so that $\langle V\rangle=0$.

\section{Methods of analysis}

Much of the scalar transport at the canopy-atmosphere interface is due to localized eddy motion in time (Raupach et al. 1996; Paw U et al. 1992; Paw U et al. 1995; Bergstrom and Hogstrom 1989); hence, a localized basis function is preferred for spectral and cospectral analysis and for characteristic length scale identification. A localized basis function in the time domain has advantages over its Fourier counterpart due to its ability to concentrate much of this energy in few wavelet coefficients (Katul and Vidakovic 1996). Hence, orthonormal wavelet transforms "disbalance" energy of many flow variables. For completeness, key derivations pertaining to wavelet spectra, cospectra, and Lorentz wavelet filtering approaches are first reviewed.

\section{a. Wavelet analysis}

Orthonormal wavelet expansions are used since the basis functions are orthogonal and mutual independence of wavelet coefficients is guaranteed at all scales. Using a logarithmic uniform spacing for the scale discretization with increasingly coarser resolution at larger scales (space discretization), a complete orthogonal wavelet basis can be constructed that permits decomposition of any discretely measured turbulent flow variable (e.g., $\left.u_{i}, c\right)$ vector $\mathbf{f}\left(x_{j}\right)$ into $N$ wavelet coefficients [where $j$ $\left.=0,1, \ldots, N-1 ;\left\langle\mathbf{f}\left(x_{j}\right)\right\rangle=0\right]$. Unlike Fourier transforms, many wavelet basis functions are available for this decomposition (see, e.g., Daubechies 1992).

\section{1) FAST WAVELET TRANSFORM (FWT)}

The Haar wavelet basis is selected for its 1) differencing characteristics, 2) locality in the time domain, 3) short support that eliminates any edge effects in the transformed series, and 4) wide use in atmospheric turbulence research (Howell and Mahrt 1994; Katul and Parlange 1994; Katul and Vidakovic 1996, 1998; Lu and Fitzjarrald 1994; Turner and Leclerc 1994; Turner et al. 1994; Mahrt and Howell 1994; Yee et al. 1996; Mahrt 1991). The Haar basis $\psi_{a, b}(x)=\left(a^{-1 / 2}\right) \psi[(x-$ $b) / a$, where $a=2^{m}$ and $b=2^{m} i$ is given by

$$
\psi(x)= \begin{cases}1, & \text { for } 0<x<1 / 2 \\ -1, & \text { for } 1 / 2 \leq x<1 \\ 0, & \text { elsewhere }\end{cases}
$$

where $i$ and $m$ are integers representing the position and scale indices, respectively. For the Haar wavelet, the coefficients $\mathrm{WC}^{(m+1)}(k)$ and the coarse-grained signal $S^{(m+1)}(k)$ (i.e., a low-pass filtered series) at scale $(m+$ 1) are computed from $S^{(m)}$ at scale $m$ using

$$
\mathrm{WC}^{(m+1)}(i)=\frac{1}{\sqrt{2}}\left[S^{(m)}(2 i-1)-S^{(m)}(2 i)\right]
$$

and

$$
S^{(m+1)}(k)=\frac{1}{\sqrt{2}}\left[S^{(m)}(2 i-1)+S^{(m)}(2 i)\right],
$$

for $m=0$ to $M-1, i=0$ to $2^{M-m-1}-1$, and $M=$ $\log _{2}(N), N\left(=2^{M}\right)$ is the number of samples. The wavelet coefficients (WCs) and coarse-grained signal $\left(S^{(m)}\right)$ are calculated from simultaneous application of (2) and (3) to the measurement vector $\left[\mathbf{f}\left(x_{j}\right)\right.$ originally stored in $\left.S^{(0)}\right]$ for $m=0$ to $M-1$, where $\left\langle S^{(0)}\right\rangle=0$. The decomposition in (2) produces $N-1$ wavelet coefficients defining the Haar wavelet transform of $\mathbf{f}\left(x_{j}\right)$ (see, e.g., Katul et al. 1994; Katul and Parlange 1994; and Katul and Vidakovic 1996).

\section{2) WAVELET SPECTRA AND COSPECTRA}

The $N-1$ discrete Haar WCs satisfy the energy conservation

$$
\sum_{j=0}^{j=N-1} f(j)^{2}=\sum_{m=1}^{m=M} \sum_{i=0}^{i=\left(2^{M-m}-1\right)}\left[\mathrm{WC}^{(m)}(i)\right]^{2},
$$

which is analogous to Parseval's identity. The total energy $T_{E}$ contained in a scale, $R_{m}$, is computed from the sum of the squared WCs at scale index $(m)$ using

$$
T_{E}\left(R_{m}\right)=\frac{1}{N} \sum_{i=0}^{i=\left(2^{M-m}-1\right)}\left(\mathrm{WC}^{(m)}[i]\right)^{2}
$$

and

$$
\left\langle S^{(0)^{2}}\right\rangle=\sum_{m=1}^{m=M} T_{E}\left(R_{m}\right),
$$

where $R_{m}=2^{m} d y$ (with $d y=f_{s}^{-1}\langle U\rangle$ ) is typically estimated using Taylor's (1938) hypothesis and later adjusted by the ratio of convective to time-averaged velocities, and $f_{s}$ is the sampling frequency $(10 \mathrm{~Hz}$ for this experiment). From this identity, the Haar wavelet power spectrum (energy per unit wavenumber) for a given angular wavenumber $K_{m}$ corresponding to scale $R_{m}$ is (Meneveau 1991)

$$
K_{m}=\frac{2 \pi}{R_{m}} .
$$

Hereafter, $K$ refers to wavenumber for representation simplicity unless the discrete $K_{m}$ definition is required in a derivation. Hence, the power spectral density function $P\left(K_{m}\right)$ is computed by dividing $T_{E}$ by the change in wavenumber $\Delta K_{m}\left(=2 \pi 2^{-\mathrm{m}} d y^{-1} \ln [2]\right)$, so that

$$
P\left(K_{m}\right)=\frac{\left\langle\left\langle\left(\mathrm{WC}^{(m)}[i]\right)^{2}\right\rangle\right\rangle d y}{2 \pi \ln (2) 2^{-m}},
$$

where $\langle\langle\cdot\rangle\rangle$ is averaging over all values of the position index $(i)$ at scale index $(m)$ (see Szilagyi et al. 1996 for 
discussion of alternative definition of $\left.\Delta K_{m}\right)$. Note that the wavelet power spectrum at wavenumber $K_{m}$ is directly proportional to the average of the squared wavelet coefficients at that scale. Because the power at $K_{m}$ is determined by averaging many squared wavelet coefficients, the wavelet power spectrum is generally smoother than its Fourier counterpart (see Katul and Parlange 1994) and does not require the usual windowing and tapering.

The Haar wavelet cospectrum of two turbulent flow variables (e.g., $u$ and $c$ ) can be calculated from their respective wavelet coefficient vectors $\left(\mathrm{WC}_{u}^{(m)}\right.$ and $\left.\mathrm{WC}_{c}^{(m)}\right)$ at wavenumber $K_{m}$ using

$$
P_{u, c}\left(K_{m}\right)=\frac{\left.\left\langle\left\langle\left(\mathrm{WC}_{u}^{(m)}[i]\right) \mathrm{WC}_{c}^{(m)}[i]\right)\right\rangle\right\rangle d y}{2 \pi 2^{-m} \ln (2)},
$$

where

$$
\langle u c\rangle=\frac{1}{N} \sum_{m=1}^{m=M} \sum_{i=0}^{i=\left(2^{M-m}-1\right)}\left\{\left[\mathrm{WC}_{u}^{(m)}(i)\right]\left[\mathrm{WC}_{c}^{(m)}(i)\right]\right\},
$$

as discussed by Hayashi (1994), Katul and Parlange (1994, 1995), Katul and Vidakovic (1996), and Yee et al. (1996). It should be noted that the above wavelet expansions preserve variances and covariances. This complete and orthogonal wavelet decomposition differs from continuous wavelet analysis used in Brunet and Collineau (1994), Collineau and Brunet (1993), Gao and Li (1993), Lu and Fitzjarrald (1994), Qiu et al. (1995), Turner and Leclerc (1994), and Turner et al. (1994), in which wavelet analysis was used to identify specific structural elements in the turbulence measurements.

\section{b. Wavelet thresholding}

In Vidakovic (1995) and Katul and Vidakovic (1996, 1998), it was demonstrated that discrete orthonormal wavelet transformation disbalances energy of turbulent flow variables but preserves their variances. That is, energy of the transformed time series is concentrated in only a few wavelet coefficients (usually less than 10\% for many turbulent flow variables). Such a disbalance between number of wavelet coefficients containing energy and dimension $(=N)$ offers a practical method to extract "active" turbulence from vertical velocity measurements. Such energy extraction is based on the premise that much of the energy in vertical velocity is due to active turbulence (Raupach et al. 1996). Extracting energy containing events from their respective wavelet coefficients can be readily achieved using Lorentz wavelet filtering discussed next. Prior to discussing Lorentz wavelet filtering, the distinction between active and inactive eddy motion is first considered.

\section{1) Active And InActive EDdy Motion}

The decomposition of eddy motion into active and inactive was first proposed by Townsend (1961) for equilibrium layers (analogous to the neutral atmospheric surface layer). Townsend's (1961) hypothesis states that eddy motion in the equilibrium layer can be decomposed into an active component, which is a function of ground shear stress $\left(u_{*}\right)$ and height $(z)$, and an inactive component, which is produced by turbulence in the outer region. The inactive component is partly produced by the irrotational motion due to pressure fluctuations and by the large-scale vorticity field in the outer layer, which the equilibrium region senses as rapid variability in the mean flow. Raupach et al. (1991) suggested that inactive eddy motion scales with $u_{*}$ and boundary layer depth (see Katul et al. 1996a for details). In the context of canopy turbulence, eddy motion responsible for much of the vertical transport is termed as active, while eddy motion with scales much larger than the canopy height are termed inactive.

\section{2) LORENTZ THRESHOLDING}

A general measure of energy disbalance for a given measurement vector in a particular domain such as time, wavelet, or Fourier $\left(\gamma_{i}, i=1,2, \ldots, N\right)$ is the Lorentz curve $L(p)$. The $L(p)$ curve is a convex curve describing the cumulative energy that is contained in $p$ smallest energy components. The $L(p)$ curve is computed by ranking, in ascending order, the energy in the wavelet coefficients and plotting the cumulative loss in energy (ordinate axis) incurred upon removing, sequentially, one coefficient until all $N$ coefficients are removed (plotted on abscissa). A sample Lorentz curve is shown in Fig. 1a for which the abscissa is the cumulative number of coefficients removed (in fraction) and the ordinate is the cumulative energy loss (in fraction). In Fig. 1a, the convexity in $L(p)$ relative to the diagonal line (ideally balanced energy) is directly proportional to energy disbalance. Note in Fig. 1a that energy disbalance in the wavelet domain is larger than its time or Fourier counterpart. Typically, high-energy disbalance in the time domain is associated with strong intermittency so that it suffices to retain only a small fraction of time intervals within a time series, while high-energy disbalance in the Fourier domain is associated with concentration of energy at low wavenumbers so that it suffices to retain only few Fourier modes. Since canopy turbulent flows are intermittent in time and exhibit energy concentration at low wavenumbers, it is natural that energy be concentrated in even fewer wavelet modes given that wavelet decompositions are sensitive to the time-frequency properties of turbulent flow variables.

The well-defined and unique inflection point in the wavelet Lorentz curve $L(p)$ suggest an objective method to threshold wavelet coefficients. The inflection point can be identified as the point for which the $L(p)$ curve slope is unity. Hence, in Lorentz thresholding, wavelet coefficients are set to zero if 
(a)

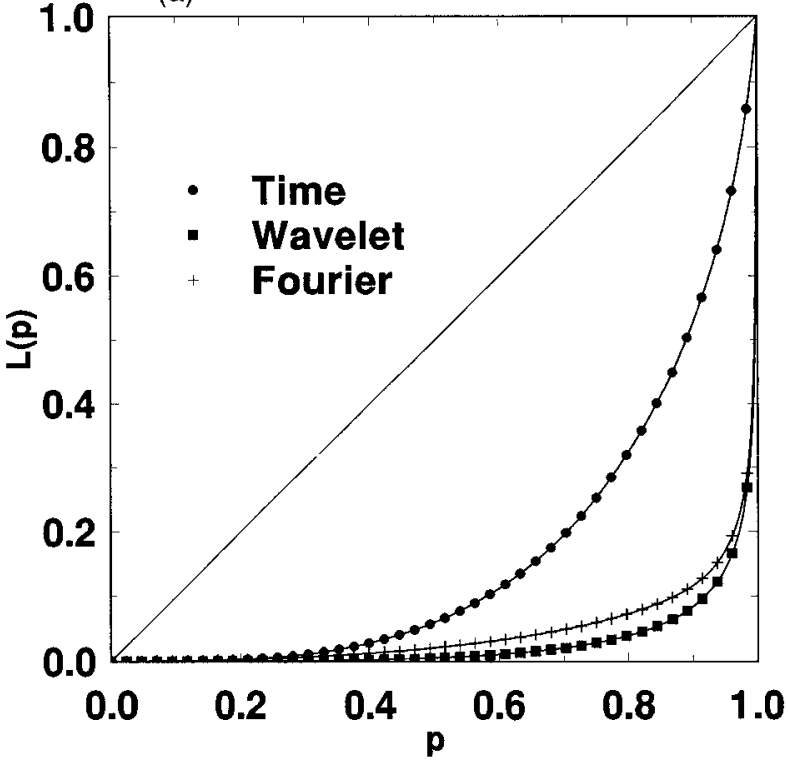

(b)

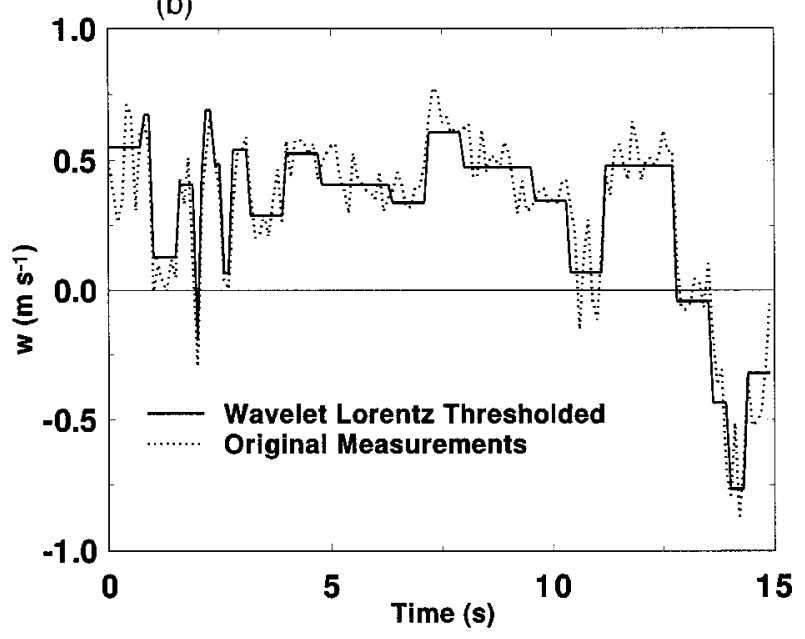

FIG. 1. (a) Sample Lorentz curves $L(p)$ in time (solid circle), Fourier (plus), and wavelet (solid rectangles) domains. The abscissa and ordinates are the cumulative coefficients (from 0 to 1 ) and the corresponding loss in vertical velocity energy (from 0 to 1 ) should these coefficients be thresholded. The point $p$ at which the tangent of $L(p)$ is unity defines the thresholding criterion $(\beta)$. All coefficients smaller than $p$ are eliminated as in (10). The solid diagonal line is for a perfectly balanced signal. The more disbalanced curve, measured by the area between the diagonal and $L(p)$, is for the wavelet domain. (b) Comparison between original and reconstructed vertical velocity time series. For clarity, only $15 \mathrm{~s}$ are shown. The run is from the hardwood forest experiment (16 June 1996).

$$
\left\{\begin{array}{l}
\mathrm{WC}_{k}^{2}<\beta_{t h}, \\
\beta_{t h}=\frac{1}{N} \sum_{k=1}^{k=N} \mathrm{WC}_{k}^{2},
\end{array}\right.
$$

where $\mathrm{WC}_{k}(k=1, \ldots, N)$ are the $N$ wavelet coefficients of some turbulent flow variable. The criterion in (10) is based on the premise that gain in parsimony by thresholding an additional coefficient is exactly balanced by loss in energy (Vidakovic 1995; Katul and Vidakovic 1996). Since active motion is the main energy contributor to $w$ (i.e., $\sigma_{w}^{2}$ ) and since Lorentz thresholding retains only large-amplitude wavelet coefficients, then the nonzero wavelet coefficients in (10) are associated with "active turbulence." In analogy to conditional sampling, it is convenient to define the relative frequency $D_{w}$ as the percent ratio of nonzero wavelet coefficients to total wavelet coefficients. In wavelet thresh- olding, $D_{w}$ is considered as a measure of compression efficiency (Farge et al. 1992).

To graphically demonstrate the effects of Lorentz wavelet thresholding on a given vertical velocity time series, Fig. 1b displays $15 \mathrm{~s}$ of measured and wavelet thresholded $w$ from a hardwood forest at $z=h$ (see Table 1). The wavelet thresholded time series is computed by wavelet transforming the original time series, applying Lorentz threshold as in (10), and finally inverse transforming the thresholded series back to the time domain. For this run, $91 \%$ of the wavelet coefficients $\left(D_{w}=0.09\right)$ were set to zero. The remaining $9 \%$, which contains $91 \%$ of the variance, was used to reconstruct the time series from the nonzero wavelet coefficients. We associate the wavelet thresholded time series with active turbulence due to arguments discussed in section 4c. We note that an earlier study by Howell and Mahrt

TABLE 1. Summary of runs, general comments on canopy morphology, leaf area index (LAI), and the range of sensible $(H)$ and latent heat (LE) fluxes during the experiment. Here, PI95 and PI96 refer to the 1995 and 1996 pine forest experiments, and HW96 and NCAR96 refer to the 1996 hardwood forest experiment with measurements collected at $z / h=1$ and measurements by NCAR collected at $z / h=1.2$.

\begin{tabular}{|c|c|c|c|c|c|c|c|}
\hline \multirow[b]{2}{*}{ Experiment } & \multirow[b]{2}{*}{ Stand } & \multirow[b]{2}{*}{ Dates } & \multirow[b]{2}{*}{ Runs } & \multicolumn{4}{|c|}{ Comments } \\
\hline & & & & $\begin{array}{c}h \\
(\mathrm{~m})\end{array}$ & LAI & $\begin{array}{c}H \\
\left(\mathrm{~W} \mathrm{~m}^{-2}\right)\end{array}$ & 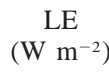 \\
\hline PI95 & Pine $(z / h=1.0)$ & 12 Aug, 1 Oct 1995 & 40 & 13 & $2.9-3.1$ & $20-296$ & $44-440$ \\
\hline PI96 & Pine $(z / h=1.0)$ & 4, 5, 9 May 1996 & 52 & 14 & 2.5 & $20-283$ & $90-455$ \\
\hline HW96 & Hardwood $(z / h=1.0)$ & 16-18 Jun; 2, 3, 8 Jul 1996 & 71 & 33 & 6.1 & $20-296$ & $85-532$ \\
\hline NCAR96 & Hardwood $(z / h=1.2)$ & 20, 24 Jun 1996 & 20 & 33 & 6.1 & $20-296$ & $85-532$ \\
\hline
\end{tabular}


(1994) established the filtering potentials of multiresolution wavelet analysis to extract energy-containing events. The above approach differs from their adaptive multiresolution filtering scheme since neither a window width (constant cutoff scale) nor a small-scale cutoff variance is needed.

\section{Experimental setup}

Eddy correlation measurements from two forest experiments at the Blackwood Division of the Duke Forest $\left(35^{\circ} 58^{\prime} \mathrm{N}, 79^{\circ} 08^{\prime} \mathrm{W}\right.$; elevation, $\left.163 \mathrm{~m}\right)$ near Durham, North Carolina, were collected and analyzed.

\section{a. Pine forest experiment}

These measurements were collected in 1995 and 1996 for a few days at different seasons. The site is a uniformly aged managed loblolly pine forest that extends $1000 \mathrm{~m}$ in the north-south direction and $600 \mathrm{~m}$ in the east-west direction. The stand, originally grown from seedlings planted at $2.4 \mathrm{~m} \times 2.4 \mathrm{~m}$ spacing in 1983 , is $14 \mathrm{~m}$ tall (the $h$ variable). Given the stand uniformity and the tree-to-tree spacing in relation to canopy height, the stand can be classified as extensive. Further details about understory species composition, soil type, and the general climatic conditions of the area can be found in Ellsworth et al. (1995), Katul et al. (1996b), Katul et al. (1997a), and Katul et al. (1997b).

Eddy correlation instruments, consisting of a Campbell Scientific Krypton $\left(\mathrm{KH}_{2} \mathrm{O}\right)$ hygrometer collocated with a triaxis Gill sonic anemometer having a path length $d_{\mathrm{sl}}=0.149 \mathrm{~m}$, were positioned on a $22-\mathrm{m}$ aluminum walk-up tower at $z / h=1$. Other details about the Gill sonic anemometer can be found in Katul et al. (1994) and Katul (1994). The sonic anemometer was positioned at $150 \mathrm{~cm}$ from surrounding leaves to avoid potential sonic wave reflection by pine needles and minimize the "wake turbulence" effects. The $U, V, W, T_{a}$, and $Q$ time series were sampled at $10 \mathrm{~Hz}$ and segmented into runs for which the sampling duration per run $\left(T_{p}\right)$ was $27.3 \mathrm{~min}$. The run fragmentation resulted in 16384 $\left(=2^{14}\right)$ measurements per flow variable per run $(M=$ 14). All analog signals from the Gill anemometer and the $\mathrm{KH}_{2} \mathrm{O}$ hygrometer instruments were sampled by a $21 \times$ Campbell Scientific micrologger and transferred to the hard drive of a personal computer via an optically isolated RS232 interface for further processing. The raw measurements for each run were then transformed so that the mean longitudinal velocity $\langle U\rangle$ was aligned along the mean horizontal wind direction and $\langle V\rangle=0$. In general, data collection started around $0900 \mathrm{LT}$ and was terminated at about 1800 LT unless storms or rain events were forecasted the previous day. The amount of runs collected each day varied from 8 to 19 runs. Time series were inspected to identify and exclude runs 1) without constant mean virtual temperature and water

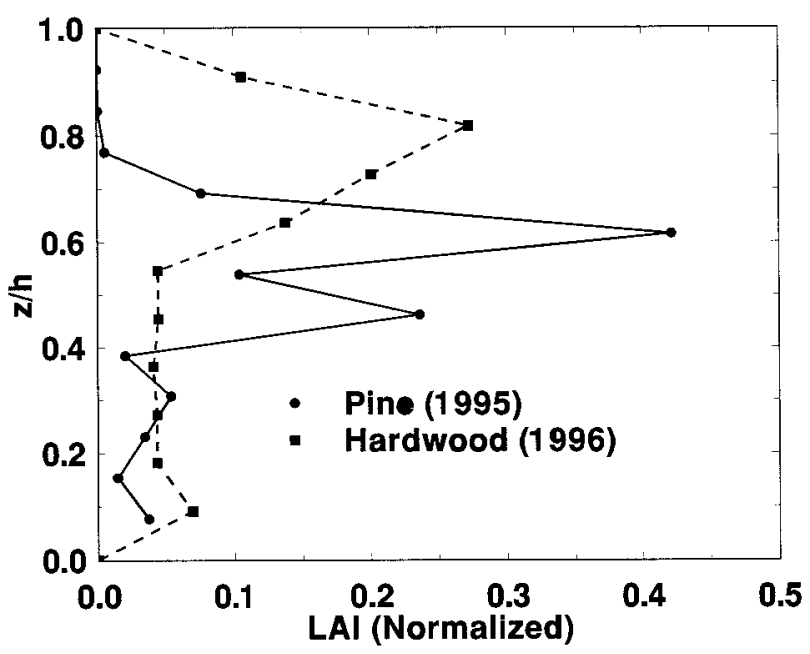

FIG. 2. Normalized leaf area index profiles. The area under both profiles is normalized to unity and the height from the ground surface is normalized by the canopy height $(h)$.

vapor concentrations and 2) with negative sensible or latent heat flux. Table 1 summarizes the runs used.

\section{b. Hardwood forest experiment}

This stand is a 33-m-tall (standard deviation in height is $8 \mathrm{~m}$ ) unevenly aged mature second growth deciduous hardwood mix with the oldest individual exceeding 180 years (Bowling et al. 1997). At this stand, a 40-m aluminum walk-up tower was used to mount eddy correlation instruments. The tower is in a flat area known as the Meadow Flats, which describes the topography for $500 \mathrm{~m}$ in all directions (Conklin 1994). About $1.1 \mathrm{~km}$ west-northwest and at $1.7 \mathrm{~km}$ northwest of the tower, the slopes of the Bald Mountains (peak = $232 \mathrm{~m}$ ) and Blackwood Mountains (peak $=226 \mathrm{~m}$ ) begin their rise, respectively. These slopes are less than $15 \%$, and their influence on the airflow close to the tower can be, in a first-order analysis, neglected (see Kaimal and Finnigan 1994, 169). The instruments were positioned on the southwest face of the tower to allow unobstructed exposure to the region's prevailing southwest winds. The site details and species composition are presented in Conklin (1994) and Katul et al. (1997b). For comparison with the pine stand, the normalized leaf area index (LAI) profiles for the two stands are shown in Fig. 2. Note that the two stands have very distinct LAI profiles in terms of leaf concentration close to the canopy top. The normalized LAI profile for each stand was measured by a LICOR 6000 LAI meter.

Datasets were collected in June-July 1996 and are described below. The first dataset was collected at $z / h$ $=1.2$ by the National Center for Atmospheric Research (NCAR) starting 20 June and ending 25 June while testing a high-frequency isoprene gas analyzer. The second dataset was collected at $z / h=1.0$ from 17 June to 
11 July using the same configuration as the pine forest experiment.

\section{1) THE $z / h=1.0$ DATASET}

In this dataset, $10-\mathrm{Hz}$ velocity, temperature, and water vapor measurements were collected using the same instruments from the pine experiment at $z / h=1.0$. These instruments were moved from the pine forest, positioned at the hardwood forest on 16 June 1996, and operated from 17 June to 11 July 1996 just before Hurricane Bertha swept through the region. The data acquisition system was started at 0900 LT and shut down at 1530 LT or prior to forecasted localized storm events. The runs used are described in Table 1.

\section{2) THE $z / h=1.2$ DATASET}

The flux system is composed of a Gill triaxial sonic anemometer, a LICOR-6262 $\mathrm{CO}_{2} / \mathrm{H}_{2} \mathrm{O}$ (LICOR, Lincoln, Nebraska) gas analyzer, a GFAS (Postfach, Germany) ozone gas analyzer, and a fast isoprene sensor based on chemiluminescence reaction between a primary alkene and ozone (see Hills and Zimmerman 1990; Guenther and Hills 1998). The instruments are described in Guenther and Hills (1998). All sensors were digitally sampled at $20.8 \mathrm{~Hz}$ and stored on a hard drive for processing (see Table 1). These measurements are used to assess the consistency of ML characteristic length scales to scalars having different sources and sinks at the forest-atmosphere interface than heat and water vapor.

\section{Results and discussion}

The results and discussion section is divided into three parts. In the first part, similarity constants are measured and compared to other experiments in order to assess whether the ML analogy explains key statistical flow properties at $z=h$. In the second part, wavelet spectra and cospectra are presented in order to identify flux transporting wavenumbers. In the third part, a linkage between such length scales, active turbulence, and organized motion is considered via Lorentz wavelet thresholding. An examination as to whether KH predicts characteristic flux-transporting eddy sizes for a wide range of scalars with different canopy sources and sinks is also discussed.

\section{a. Flow statistical properties}

The flow statistics available for comparison with many canopy-turbulence experiments include velocity statistics and integral length scales. For the pine and hardwood forests, these flow statistics are computed for each run and discussed next.

\section{1) Velocity statistics}

If the canopy turbulence at $z=h$ is to be universal and independent of canopy morphology (for horizontally extensive and uniform canopies), then the flow statistics described by $\beta_{i}=a_{i} \alpha$ with $\beta_{i}\left(=\langle U\rangle, \sigma_{u}\right.$, and $\sigma_{w}$ for $i=1,2$, and 3 , respectively) and $\alpha=u_{*}$ should result in constant $a_{i}(i=1,2$, and 3$)$ for both stands. Here, $\sigma_{u}\left(=\left\langle u^{2}\right\rangle^{1 / 2}\right)$ and $\sigma_{w}\left(=\left\langle w^{2}\right\rangle^{1 / 2}\right)$ are the longitudinal and vertical velocity standard deviations, respectively. The $\beta_{i}=a_{i} \alpha$ relationships are shown in Fig. 3a for all runs with the regression statistics for solid lines shown in Table 2. From Fig. 3a and Table 2 it is evident that canopy morphology details (e.g., height and foliage distribution) have minor influence on $a_{i}$ for $i=3$, which is in agreement with Raupach et al. (1996). Also, $a_{3}$ is better described by ML statistics than $a_{2}$, suggesting that the ML analogy is suitable for vertical but not horizontal transport. However, unlike Raupach et al.'s (1996) atmospheric stability argument, the canopy stability parameter $h / L_{\text {mo }}$ does influence $\sigma_{w} / u_{*}$ as evidenced in Fig. 3b, where $L_{\text {mo }}$ is the Obukhov length. In this figure, the function

$$
\frac{\sigma_{w}}{u_{*}}=1.1\left(1-\frac{h}{L_{\mathrm{mo}}}\right)^{1 / 3} \text {, }
$$

where

$$
L_{\mathrm{mo}}=-\frac{u_{*}^{3}}{k g \frac{\langle w T\rangle}{\left\langle T_{a}\right\rangle}}
$$

describes the measurements well. For small $-h / L_{\mathrm{mo}}$ $(<0.1), \sigma_{w} / u_{*}$ is consistent with its mixing layer value $(=1.1)$. However, when $-h / L_{\mathrm{mo}}$ is not small $(>1.0), \sigma_{w} /$ $u_{*}$ can be larger than its surface layer value $(=1.25)$. Furthermore, for large $-h / L_{\text {mo }}$, the $1 / 3$ free-convective power law is approached. This result is consistent with Shaw et al. (1988) and Leclerc et al. (1990), who demonstrated that thermal effects, which enhance the production of turbulent kinetic energy, are largest in the upper third of the canopy where the radiation load is highest. To further examine the ML analogy for vertical transport, integral timescales are considered next.

\section{2) INTEGRAL TIMESCALE OF VERTICAL VELOCITY}

The integral timescale $\left(I_{w}\right)$, defined as

$$
I_{w}=\int_{0}^{\infty} \rho_{w}(\tau) d \tau,
$$

where $\rho_{w}(\tau)$ is the vertical velocity autocorrelation function and $\tau$ is time lag, can be related to the characteristic frequency $\left(f_{p}\right)$ of KH waves predicted from HST. Using the "generic" canopy length scale parameters from Raupach et al. (1996), assuming that eddy convection ve- 
(a)

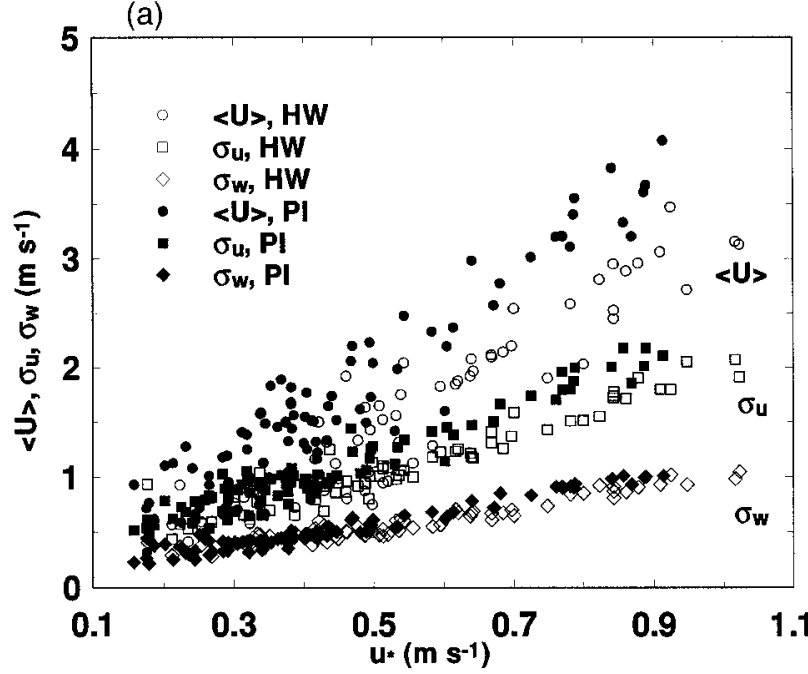

(c)

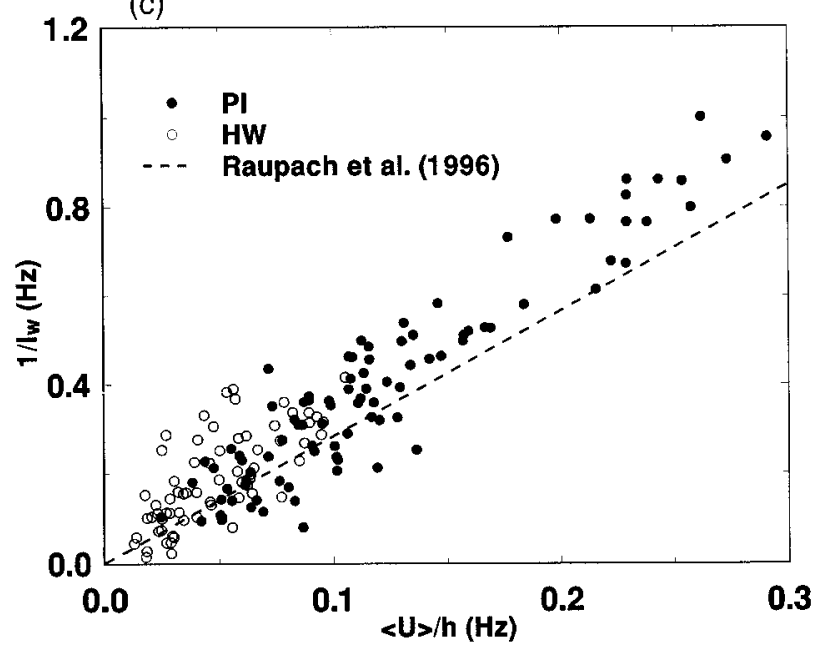

(b)

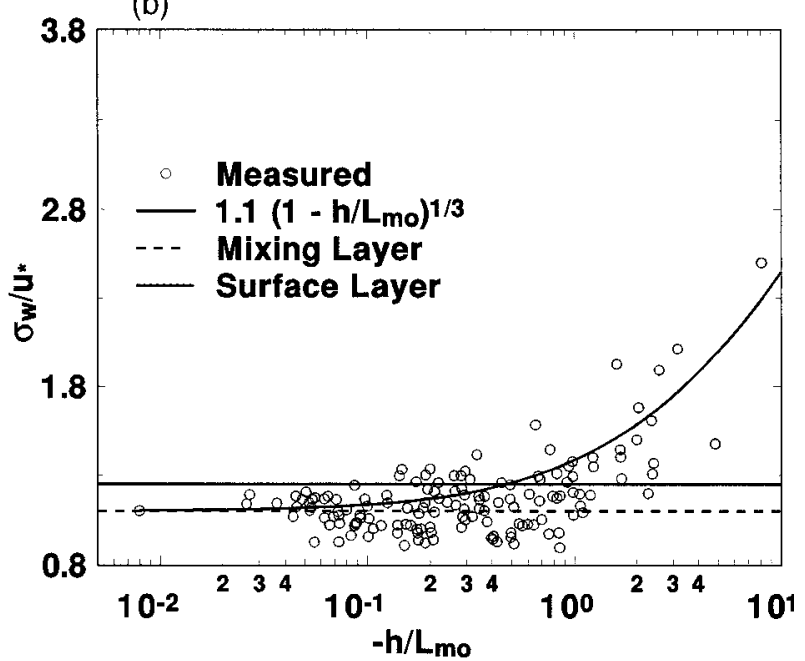

FIG. 3. (a) Flow statistical properties $\langle U\rangle, \sigma_{u}$, and $\sigma_{w}$ as a function of $u_{*}$ for both stands. (b) The influence of canopy stability function on $\sigma_{w} / u_{*}$. For neutral mixing and surface layers, $\sigma_{w} / u_{*}=1.1$ and 1.25 , respectively. (c) The variation of $I_{w}^{-1}$ with $\langle U\rangle / h$. The solid line is derived by us from Raupach et al.'s (1996) generic canopy data.

TABLE 2. Comparison between measured flow statistics from this experiment, previously published similarity constants for the canopy sublayer (CSL) from Raupach et al. (1996), and similarity constants measured in laboratory mixing layers (MLs). The regression statistics for the model $\beta=a \alpha$ are presented. The coefficient of determination $\left(R^{2}\right)$, the standard error of estimate (SEE), and the standard error in a coefficient (SEC) are also shown. The $N_{w} / T_{p}$ regression analysis resulted in significant intercepts.

\begin{tabular}{|c|c|c|c|c|c|c|c|}
\hline Flow variable $(\beta)$ & $\alpha$ & $a$ & SEE & $R^{2}$ & SEC & CSL & ML \\
\hline$\langle U\rangle$ (Overall) & $u_{*}$ & 3.54 & $0.36 \mathrm{~m} \mathrm{~s}^{-1}$ & 0.85 & 0.06 & $2.4-5.0$ & $3.6^{\mathrm{a}}$ \\
\hline$\langle U\rangle$ (Pine) & & 3.87 & $0.31 \mathrm{~m} \mathrm{~s}^{-1}$ & 0.88 & 0.07 & & \\
\hline$\langle U\rangle$ (Hardwood) & & 2.97 & $0.32 \mathrm{~m} \mathrm{~s}^{-1}$ & 0.84 & 0.07 & & \\
\hline$\sigma_{u}($ Overall $)$ & $u_{*}$ & 2.25 & $0.17 \mathrm{~m} \mathrm{~s}^{-1}$ & 0.85 & 0.03 & 1.8 & 1.7 \\
\hline$\sigma_{u}($ Pine $)$ & & 2.40 & $0.13 \mathrm{~m} \mathrm{~s}^{-1}$ & 0.90 & 0.03 & & \\
\hline$\sigma_{u}($ Hardwood $)$ & & 2.02 & $0.14 \mathrm{~m} \mathrm{~s}^{-1}$ & 0.87 & 0.01 & & \\
\hline$\sigma_{w}($ Overall $)$ & $u_{*}$ & 1.11 & $0.07 \mathrm{~m} \mathrm{~s}^{-1}$ & 0.90 & 0.01 & 1.1 & 1.3 \\
\hline$\sigma_{w}($ Pine $)$ & & 1.16 & $0.06 \mathrm{~m} \mathrm{~s}^{-1}$ & 0.91 & 0.01 & & \\
\hline$\sigma_{w}($ Hardwood $)$ & & 1.05 & $0.07 \mathrm{~m} \mathrm{~s}^{-1}$ & 0.87 & 0.01 & & \\
\hline$I_{w}^{-1}$ & $\langle U\rangle / h$ & 3.3 & $0.08 \mathrm{~s}^{-1}$ & 0.87 & 0.11 & $2.84^{\mathrm{b}}$ & \\
\hline$N_{w} / T_{p}^{\mathrm{c}}$ & $\langle U\rangle / h$ & 0.65 & $0.02 \mathrm{~s}^{-1}$ & 0.81 & 0.026 & 0.45 & \\
\hline$N_{w} / T_{p}^{\mathrm{d}}$ & $I_{w}^{-1}$ & 0.194 & $0.018 \mathrm{~s}^{-1}$ & 0.85 & 0.026 & 0.16 & \\
\hline
\end{tabular}

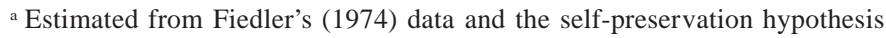

${ }^{\mathrm{b}}$ Estimated from Raupach et al.'s (1996) generic canopy parameters.

${ }^{c}$ The regression intercept $=0.066$ is significant.

${ }^{\mathrm{d}}$ The regression intercept $=0.067$ is significant . 
(a)

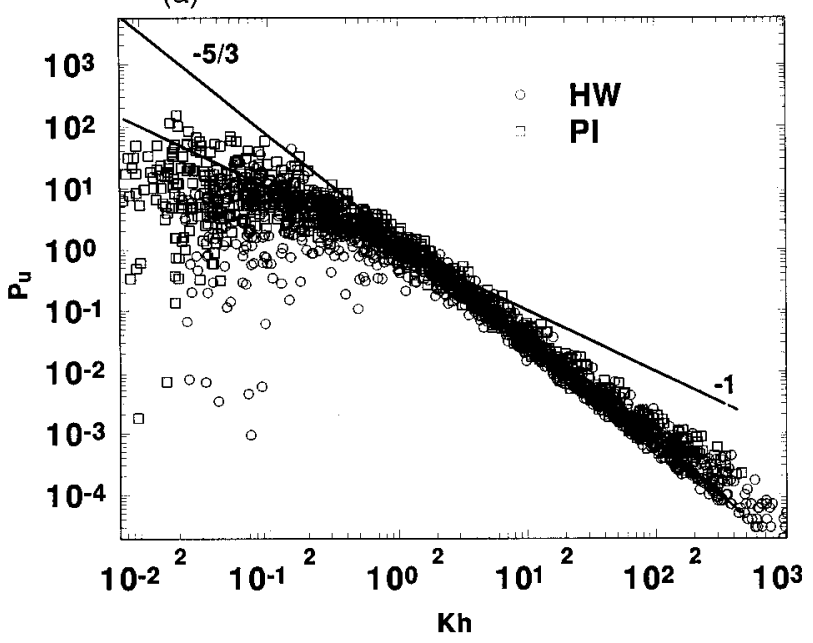

(c)

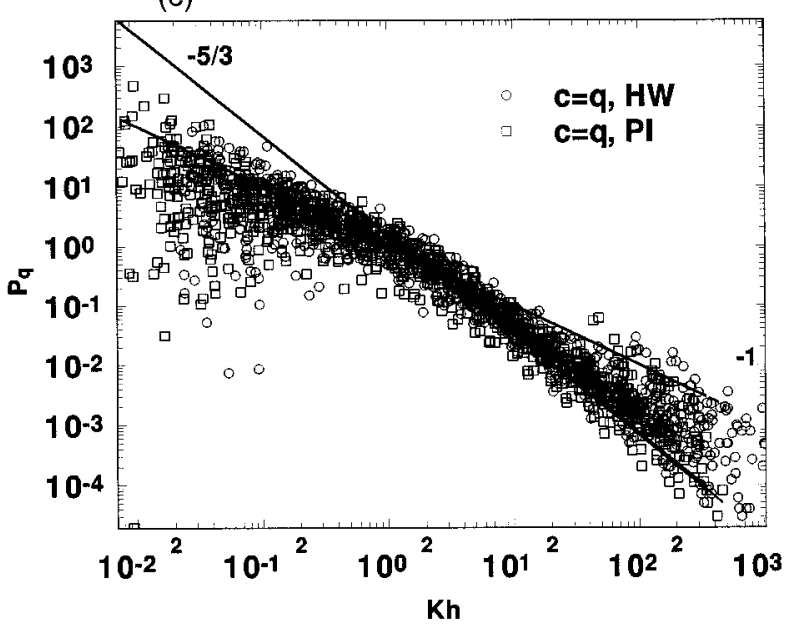

locity $\left(U_{c}\right)$ is $1.8\langle U\rangle$ from Shaw et al. (1995), noting that $f_{p}=U_{c} / \Lambda_{x}$, and after some algebra, we obtain

$$
\frac{1}{I_{w}}=2.84 \frac{\langle U\rangle}{h} .
$$

We decided to use the ratio $U_{c} /\langle U\rangle=1.8$ found experimentally by Shaw et al. (1995) in order to compare with Raupach et al.'s (1996) generic canopy parameters. Furthermore, much of the theoretical results relating $U_{c}$ to $\langle U\rangle$ as in Lumley (1965), Wyngaard and Clifford (1977), and Hill (1996) assume low squared turbulent intensity (i.e., $\left.\left[\sigma_{u} /\langle U\rangle\right]^{2}<0.1\right)$. For our experiment, the squared turbulent intensity varied from 0.2 to 2 ; hence, the application of such theoretical models introduces large uncertainties. From (12), $I_{w}$ is calculated for all runs (integration is terminated at the first zero crossing, as discussed in Katul et al. 1997a) and compared to $\langle U\rangle /$ $h$ in Fig. 3c. The regression model $I_{w}^{-1}=a_{4}\langle U\rangle / h$ is used, as before, to estimate $a_{4}$ from measurements in Fig. 3c (see Table 2 for regression statistics). Given that the "generic canopy" of Raupach et al. (1996) assumes (b)

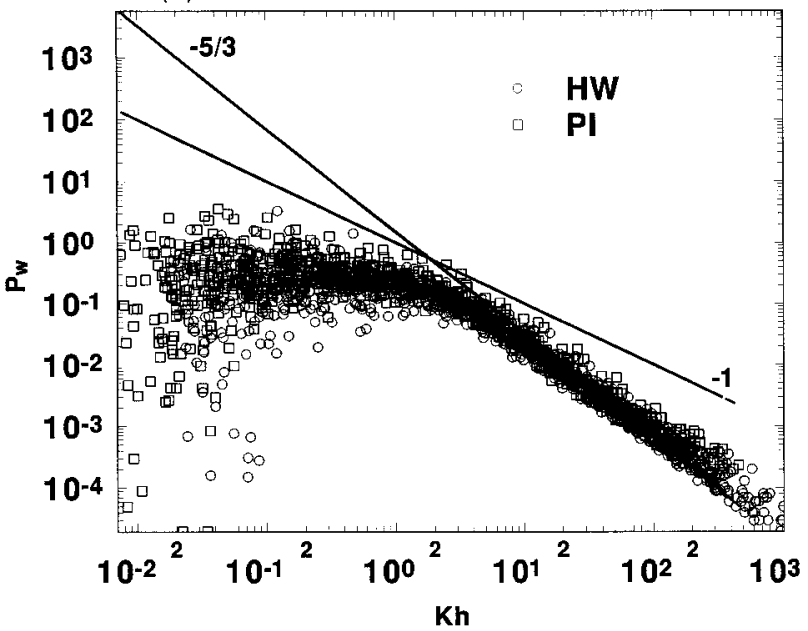

FIG. 4. Normalized wavelet power spectra as a function of normalized wavenumbers $(K h)$ for (a) $u$, (b) $w$, and (c) $q$ for all 159 runs. The -1 and $-5 / 3$ power laws are also shown (solid lines).

that the shear length scale $\left(L_{s}\right)$ defined as $\langle U\rangle(d\langle U\rangle /$ $d z)^{-1}$ is $h / 2$, the measured $a_{4}=3.3$ is in good agreement with HST predictions. This agreement demonstrates that vertical transport is controlled by active turbulence at $z$ $=h$. To further examine the statistical properties of active turbulence, wavelet spectra and cospectra are considered next.

\section{b. Wavelet analysis}

\section{1) WAVELET ENERGY AND POWER SPECTRA}

In Figs. $4 \mathrm{a}-\mathrm{c}$ the dimensionless wavelet power spectra of $u\left(=P_{u}\right), w\left(=P_{w}\right)$, and $c\left(=P_{c}, c=q\right.$ as an example $)$ are shown along with the -1 and $-5 / 3$ power laws for both forest stands, respectively. The wavelet spectra are normalized with characteristic velocity, scalar, and length scales corresponding to $u_{*}, c_{*}\left(=\langle w c\rangle / u_{*}\right)$, and $h$. The wavenumber, computed from Taylor's (1938) frozen turbulence hypothesis and adjusted by $1.8\left(=U_{c} /\right.$ $\langle U\rangle)$, is normalized by $h$. Note from Figs. 4a,b that for $\mathrm{Kh}<1$, a -1 power law is evident in $P_{u}$ but not $P_{w}$. 


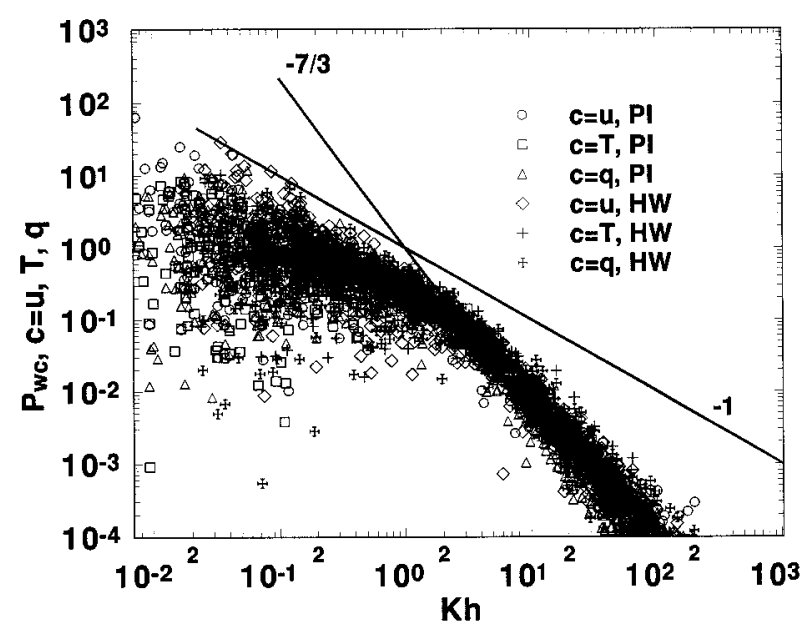

FIG. 5. Normalized wavelet power cospectra as a function of normalized wavenumbers $(K h)$ for $(u w),(w T)$, and $(w q)$. The -1 and $-7 / 3$ power laws are shown.

As discussed in Katul et al. (1996a), a - 1 power law in the $u$ spectrum at a low wavenumber is a signature of inactive eddy motion. We note that the spectra in Figs. 4a,b suggest that inactive eddy motion contributes to $\sigma_{u}^{2}$ but not $\sigma_{w}^{2}$ at $z=h$ and for both stands. While the -1 power law reflects inactive eddy motion (which scales with boundary layer height), the -1 power law in Fig. 4a does not extend beyond $K h<0.003$ because of buoyancy effects. Evidence of the -1 power law in the scalar time series in Fig. 4c also suggests that inactive eddy motion contributes to scalar variance (see Kader and Yaglom 1991).

\section{2) WAVELET POWER COSPECTRA OF VERTICAL FLUXES}

In Fig. 5, the wavelet power cospectra of the normalized $u w\left(=P_{u w}\right), w T\left(=P_{w T}\right)$, and $w q\left(=P_{w q}\right)$ are shown for all runs. Note that for $K h>2$, an approximate $-7 / 3$ power law (regression slope $=-2.08$ ), typically observed in the inertial subrange (fine scales) of surface layer turbulence, is present for nearly three decades. For $K h<1$, an approximate -1 power law is apparent at $z=h$, which is in agreement with many surface layer experiments (Kader and Yaglom 1991). The transition in power laws from -1 to $-7 / 3$ occurs at about $K h=$ 2 , which is also the "active" scale for scalar and momentum transport, as evidenced by the energy cospectra in Fig. 6. In Fig. 6, the momentum and flux-transporting eddies occur at about $K h=1.5$ and can be compared to ML predictions. As discussed in Raupach et al. (1996), the peak frequency $\left(f_{w p}\right)$ in the $w$ spectrum occurs at $f_{w p}=0.45\langle U\rangle / h$ (for a generic canopy). For $K h$ $=1.5$, the peak frequency in the flux wavelet cospectra of Fig. 6 is $1.5 \times[1.8 /(2 \pi)]\langle U\rangle / h$ (i.e., $=0.43\langle U\rangle / h$ ) and is in excellent agreement with Raupach et al. (1996). Interestingly, Figs. 5 and 6 suggest some cospectral sim-

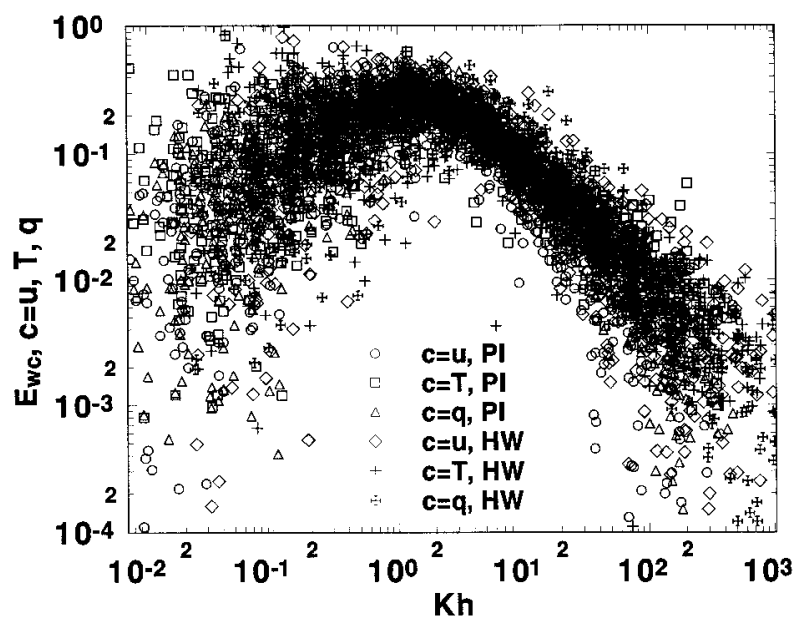

FIG. 6. Normalized wavelet energy cospectra as a function of normalized wavenumbers $(K h)$ for $u w, w T$, and $w q(z / h=1.0)$. The peak scale corresponds to $0.5 \mathrm{~h}$.

ilarity between momentum and scalar transport at both stands despite differences in canopy morphology and vertical variability in scalar and momentum sources and sinks. This point is examined using the scalar measurements at $z / h=1.2$ by NCAR in Fig. 7 . These cospectral measurements suggest that peak cospectral frequencies for $T, q, \mathrm{O}_{3}$, and $\mathrm{I}_{\mathrm{s}}$ are similar though correlation coefficients between such scalar fluctuation time series is far from unity for many runs (not shown here).

\section{c. Wavelet thresholding, organized structures, and active turbulence}

Wavelet analysis enables the estimation of $\Lambda_{x} / h$ from

$$
\frac{\Lambda_{x}}{h}=\frac{T_{p}}{N_{w}} \frac{U_{c}}{h},
$$

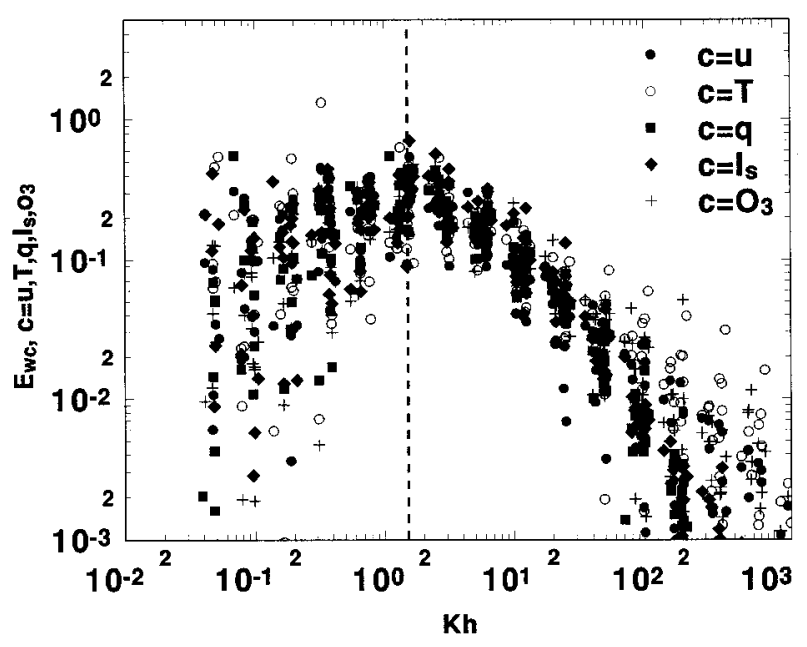

FIG. 7. Similar to Fig. 6 but at $z / h=1.2$ and for $w u, w T, w q$, $w \mathrm{O}_{3}$, and $\left(w \mathrm{I}_{\mathrm{s}}\right)$. 
(a)

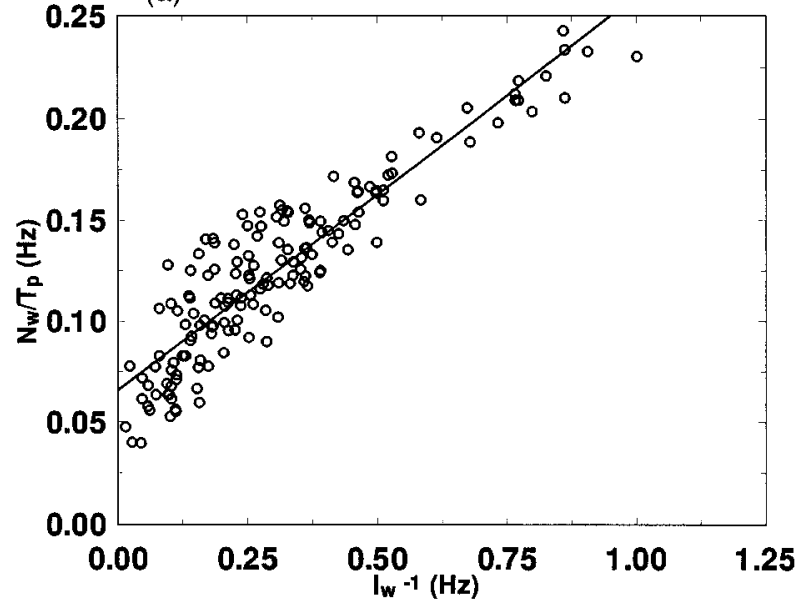

(c)

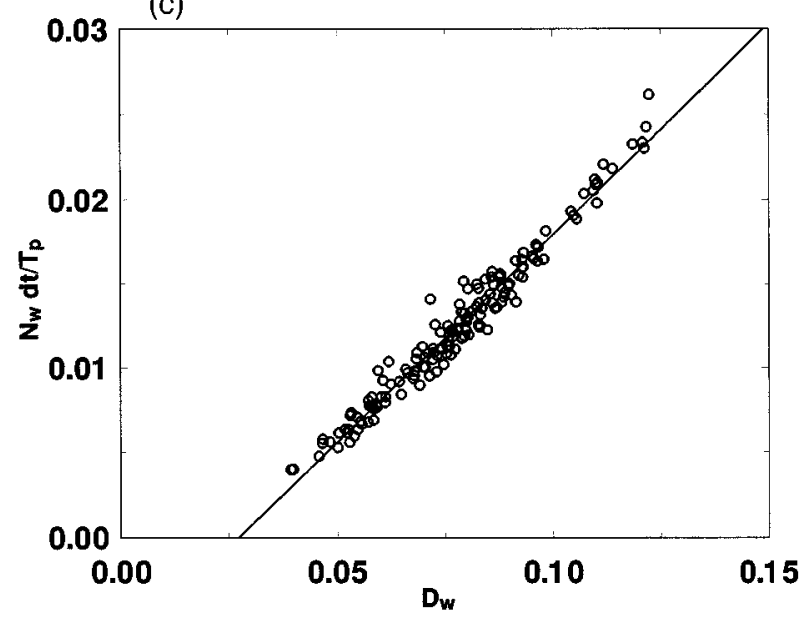

where $T_{p}$ is the sampling period $(=1638.4 \mathrm{~s}$ in this study) and $N_{w}$ is the total number of organized structures detected in $T_{p}$. The estimation $\Lambda_{x} / h$ from such an approach requires an objective and ambiguous method to estimate $N_{w} / T_{p}$. Here, we demonstrate that Lorentz wavelet thresholding can be used to estimate $N_{w} / T_{p}$ while preserving energy, covariances, and two-point statistics expected in canopy mixing layers. The approach is based on the premise that $w$ time series reconstructed from nonzero wavelet coefficients encompass only active turbulence associated with coherent structures. Hence, $N_{w} / T_{p}$ can be readily computed from counting zero crossings in the time domain. For each run, the $w$ time series is transformed to the wavelet domain, Lorentz thresholded, and transformed back to the time domain from nonzero wavelet coefficients. On average, two sequential zero crossings in the time domain describe an organized structure.

An independent test, for which the thresholded vertical velocity time series reconstructs active turbulence properties for each run, can be performed via conservation of energy and preservation of scalar mass fluxes. (b)

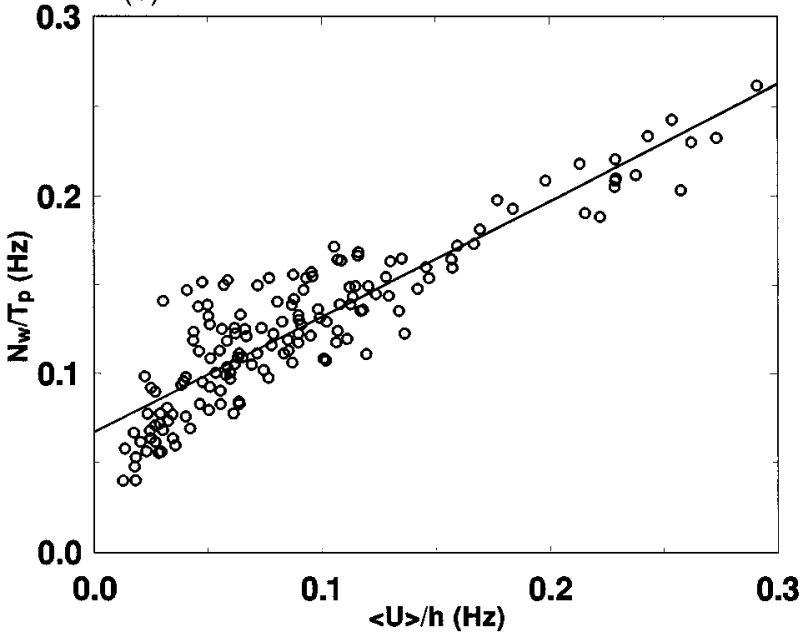

FIG. 8. (a) Variation of organized motion frequency $N_{w} / T_{p}$ with integral timescale $\left(I_{w}\right)$. The regression line (solid) is $N_{w} / T_{p}=0.194$ $I_{w}^{-1}+0.066$. (b) Same as (a) but with $\langle U\rangle / h$. The regression line (solid) is $N_{w} / T_{p}=0.65\langle U\rangle / h+0.066$. (c) Variation of the organized motion relative frequency $\left(N_{w} d t / T_{p}\right)$ with fraction of nonzero wavelet coefficients $\left(D_{w}\right)$.

Both of these tests are examined in the appendix. From the appendix, it is clear that the small number of nonzero wavelet coefficients conserves much of the variances and covariances for all runs. That $N_{w} / T_{p}$ reproduces much of the KH organized eddy motion dynamics can be tested by analytically relating $N_{w} / T_{p}$ to $I_{w}$ using

$$
\frac{\Lambda_{x}}{h}=\frac{T_{p}}{N_{w}} \frac{U_{c}}{h}=2 \pi \frac{I_{w}\langle U\rangle}{h} \frac{U_{c}}{\langle U\rangle} \Rightarrow \frac{N_{w}}{T_{p}}=\frac{1}{2 \pi I_{w}} .
$$

That is, if the time series constructed from nonzero wavelet coefficients represents active turbulence, then $N_{w} / T_{p}$ measured by zero crossings in the time domain must be $\approx 0.16 I_{w}^{-1}$. This relationship is directly tested in Fig. 8a with good agreement between zero-crossing $N_{w} / T_{p}$ predictions and measurements. Regression analysis (solid line) between $N_{w} / T_{p}$ and $I_{w}^{-1}$ resulted in a slope equal to 0.194 (see Table 2), which is close to 0.16 obtained from Raupach et al.'s (1996) generic canopy parameters and the ML analogy. Hence, such an agreement demonstrates that the proposed method for estimating $N_{w} / T_{p}$ is consistent with ML predictions. A 
similar analysis between $N_{w} / T_{p}$ and $\langle U\rangle / h$ as in (14) is shown in Fig. 8b. In comparison with other filtering approaches, Lorentz wavelet thresholding can be viewed as "data adaptive" since more wavelet coefficients are thresholded with increasing integral timescale (or $N_{w} /$ $T_{p}$ ). Such data adaptation is demonstrated by the relationship between $D_{w}$ and the time fraction of structures detected $\left(=N_{w} d t / T_{p}\right)$ using zero crossings in Fig. 8c. The relationship in Fig. $8 \mathrm{c}$ is consistent with the working hypothesis that nonzero wavelet coefficients represent organized structures. It should be noted that our proposed wavelet thresholding method differs from previous methods since the "filtering" criterion requires no calibration and no a priori structural geometry specification.

\section{Conclusions}

Turbulent velocity and scalar fluctuation measurements at the canopy-atmosphere interface of two morphologically distinct forests were collected and analyzed using the mixing layer (ML) analogy proposed by Raupach et al. (1989) and Raupach et al. (1996). We demonstrated the following.

1) The vertical velocity statistics are insensitive to canopy morphology when compared to the mean horizontal velocity $(\langle U\rangle)$. In particular, the relationship between $\langle U\rangle$ and $u_{*}$ varied by $30 \%$ from stand to stand.

2) The ML analogy predicts well the relationship between $\sigma_{w}$ and $u_{*}$. However, $\sigma_{w} / u_{*}$ diverges from its ML value $(=1.1)$ due to variations in the canopy stability parameter $\left(-h / L_{\mathrm{mo}}\right)$. When $-h / L_{\mathrm{mo}}>2$, the $1 / 3$ power law, typically associated with the free convective limit, is evident in the $\sigma_{w} / u_{*}$ measurements.

3) Using the ML analogy, the integral timescale $\left(I_{w}\right)$ is related to the arrival frequency of organized eddies $(=\langle U\rangle / h)$ whose origin is the transverse $\mathrm{KH}$. This relationship was found to not be sensitive to stability variation in agreement with a previous study by Irvine and Brunet (1996). The derived relationship from this experiment $I_{w}^{-1}=3.3\langle U\rangle / h$ is in excellent agreement with values reported by Raupach et al. (1996) for a generic canopy. A direct consequence of this result is that for tall canopies $(>10 \mathrm{~m})$, the arrival frequency is small $\left(\langle U\rangle \sim 1 \mathrm{~m} \mathrm{~s}^{-1}, I_{w}^{-1}=0.3\right.$ $\mathrm{Hz})$. When considered in concert with the rapid decay $\left(P_{w c} \sim K^{-7 / 3}\right)$ of the scalar cospectrum $\left(P_{w c}\right)$, this analysis suggests that slower response sensors having a response frequency at least an order of magnitude larger than $I_{w}^{-1}(\sim 3 \mathrm{~Hz})$ can be utilized in eddy correlation flux calculations.

4) Wavelet power spectra revealed that inactive eddy motion, in the sense of Townsend (1976), contaminates horizontal velocity and scalar fluctuations but not vertical velocity fluctuations. Since inactive eddy motion is a boundary layer phenomenon, this study demonstrates that ML is only applicable to vertical turbulent transport and flow statistics.

5) Wavelet energy cospectra revealed similarity in eddy sizes responsible for much of the scalar and momentum covariances at dimensionless wavenumber $K h=1.5$. We showed that these cospectral wavenumbers are associated with energy containing eddies of vertical velocity perturbations in agreement with the ML analogy.

6) Using Lorentz wavelet thresholding, the detection frequency of organized motion $\left(N_{w} / T_{p}\right)$ predicted from vertical velocity time series was found to be linearly related to $\langle U\rangle / h$ and $I_{w}^{-1}$ in agreement with the ML analogy. The fact that this finding agrees with a previous result by Collineau and Brunet (1993), which utilized an entirely different wavelet thresholding approach, confirms the robustness of wavelet techniques to detecting organized flow properties.

Acknowledgments. The authors would like to thank Joselyn Fenstermacher and Debbie Engel for their help in setting up the instruments at the hardwood site in 1996, Judd Edeburn and the Duke Forest staff for their general assistance at the Duke Forest, David Ellsworth for providing us with the leaf area index measurements, and George Hendrey for his support during the 199596 Pine Forest Experiment. This project was funded, in part, by the U.S. Department of Energy (DOE) through the Southeast Regional Center at the University of Alabama, Tuscaloosa (DOE Cooperative agreement DEFC03-90ER61010), and through the FACE-FACTS project (DE-FG05-95ER62083), the National Science Foundation (NSF Grants BIR-12333 and DMS9626159), and the U.S. Environmental Protection Agency (EPA Grant 91-0074-94).

\section{APPENDIX}

\section{Active Turbulence Identification with Lorentz Thresholding}

In this appendix, it is demonstrated that nonzero wavelet coefficients, obtained following Lorentz thresholding and used to reconstruct vertical velocity time series and estimate $N_{w} / T_{p}$, are associated with active turbulence. Since active turbulence is responsible for much of $\sigma_{w}^{2}$ and $\langle w c\rangle(c=T, q$, etc. ...), then the reconstructed vertical velocity time series $\left(w^{f}\right)$ variance and covariance with a scalar (i.e., $\left.\left\langle w^{f} c\right\rangle\right)$ must be preserved. We tested this hypothesis by

1) wavelet transforming the original vertical velocity time series for each run,

2) Lorentz thresholding wavelet coefficients using the criterion in (10),

3) reconstructing the vertical velocity time series from nonzero wavelet coefficients for each run $\left(w^{f}\right)$,

4) comparing variances of thresholded $\left(w^{f}\right)$ and original 
(a)

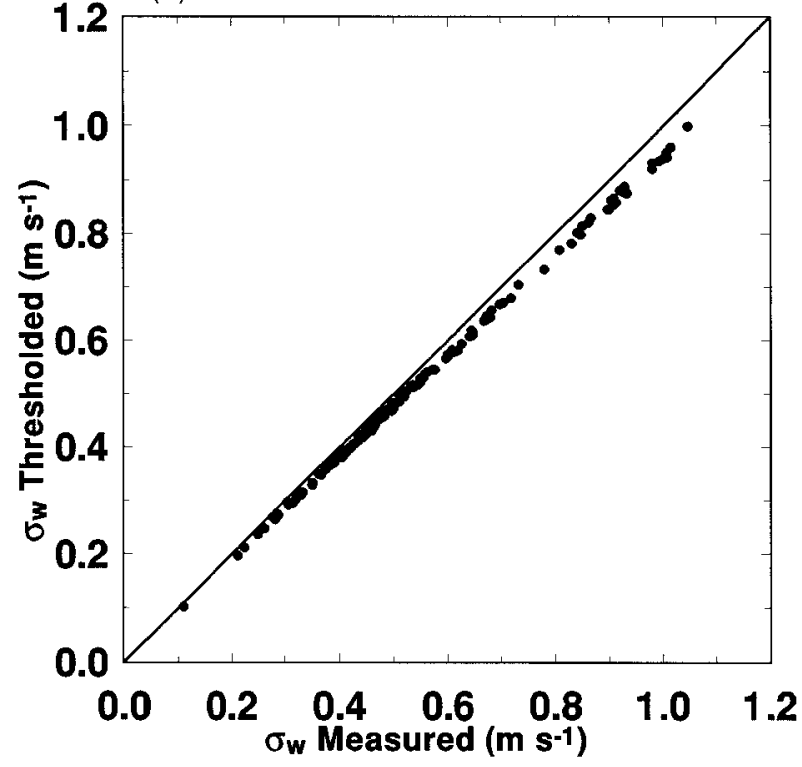

(b)

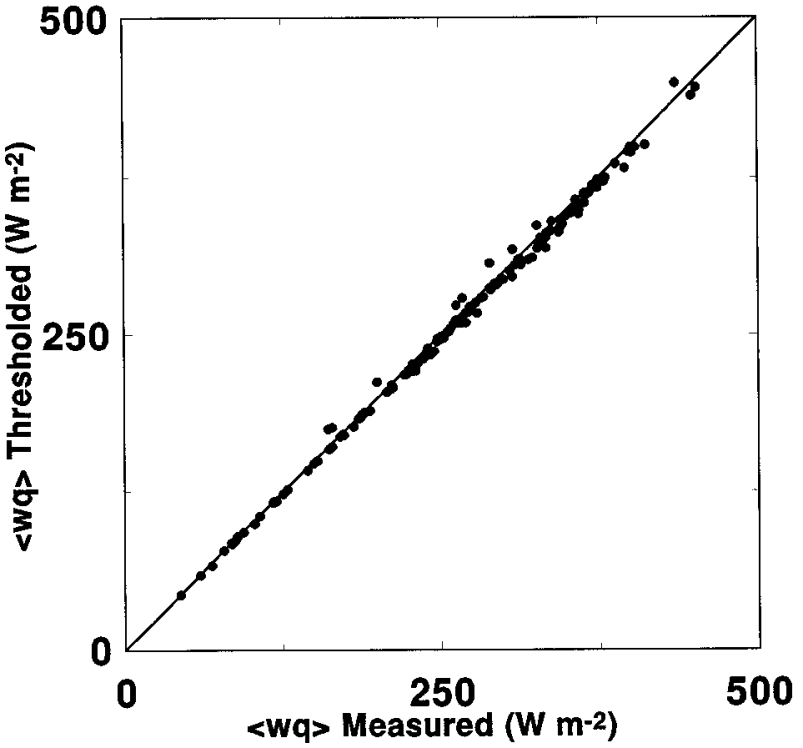

FIG. A1. (a) Comparison between thresholded and original vertical velocity standard deviations for all runs. The 1:1 line is also shown. (b) Comparison between latent heat flux $\left(\mathrm{W} \mathrm{m}^{-2}\right)$ computed from thresholded and original vertical velocity time series for all runs. The 1: 1 line is also shown.

(i.e., nonthresholded) vertical velocity time series for each run, and

5) comparing covariances $\langle w c\rangle$ and $\left\langle w^{f} c\right\rangle$.

The variance and covariance comparisons of steps (4)(1) are shown in Figs. A1a,b ( $c=q$ for illustration). From Figs. A1a,b, it is evident that few wavelet coefficients reproduce much of the variances and covariances demonstrating that less than $10 \%$ (Fig. 8c) of the wavelet coefficients are associated with active turbulence.

\section{REFERENCES}

Amiro, B. D., 1990: Comparison of turbulence statistics within three boreal forest canopies. Bound.-Layer Meteor., 51, 99-121.

— , and P. A. Davis, 1988: Statistics of atmospheric turbulence within a natural black spruce forest canopy. Bound.-Layer Meteor., 44, 267-283.

Baldocchi, D., and T. P. Meyers, 1988: Turbulence structure in a deciduous forest. Bound.-Layer Meteor., 43, 345-364.

- , and -1989 : The effects of extreme turbulent events of the estimation of aerodynamic variables in a decidous forest canopy. Agric. For. Meteor., 48, 117-134.

Bergstrom, H., and U. Hogstrom, 1989: Turbulent exchange above a pine forest. II: Organized structures. Bound.-Layer Meteor., 49, 231-263.

Bowling, D. R., A. A. Turnispeed, A. C. Delany, D. D. Baldocchi, J. P. Greenberg, and R. K. Monson, 1997: The use of relaxed eddy accumulation to measure biosphere-atmosphere exchange of isoprene and other biological trace gases. Oecologia, in press.

Brunet, Y., and S. Collineau, 1994: Wavelet analysis of diurnal and nocturnal turbulence above a maize crop. Wavelets in Geophysics, E. Foufoula-Georgiou and P. Kumar, Eds., Academic Press, 129-150.

Collineau, S., and Y. Brunet, 1993: Detection of turbulent coherent motion in a forest canopy: Wavelet analysis. Bound.-Layer Meteor., 65, 357-379.
Conklin, P., 1994: Turbulent wind, temperature, and pressure in a mature hardwood canopy. Ph.D. dissertation, Duke University, 105 pp. [Available from Duke Perkins Library, Durham, NC 27708.]

Daubechies, I., 1992: Ten Lectures on Wavelets. CBMS-NSF Regional Conf. Series in Applied Mathematics, Vol. 61, S.I.A.M., $357 \mathrm{pp}$.

Denmead, O. T., and E. F. Bradley, 1985: Flux-gradient relationships in a forest canopy. The Forest-Atmosphere Interaction, B. A. Hutchison and B. B. Hicks, Eds., D. Reidel Publishing, 421-442.

Ellsworth, D. S., R. Oren, C. Huang, N. Phillips, and G. Hendrey, 1995: Leaf and canopy responses to elevated $\mathrm{CO}_{2}$ in a pine forest under free-air $\mathrm{CO}_{2}$ enrichment. Oecologia, 104, 139-146.

Farge, M., E. Goirand, Y. Meyer, F. Pascal, and M. V. Wickerhauser, 1992: Improved predictability of two-dimensional turbulent flows using wavelet packet compression. Fluid Dyn. Res., 10, 229-250.

Fiedler, H. E., 1974: Transport of heat across a plane turbulent mixing layer. Advances in Geophysics, Vol 18A, Academic Press, 93109.

Gao, W., and B. L. Li, 1993: Wavelet analysis of coherent structures at the atmosphere-forest interface. J. Appl. Meteor., 32, 17171725 .

_- R. H. Shaw, and K. T. Paw U, 1992: Conditional analysis of temperature and humidity microfronts and ejection/sweep motions within and above a deciduous forest. Bound.-Layer Meteor., 59, 35-57.

Guenther, B. A., and A. J. Hills, 1998: Eddy covariance measurement of isoprene fluxes. J. Geophys. Res., 103, 13 145-13 152.

Hayashi, T., 1994: An analysis of wind velocity fluctuations in the atmospheric surface layer using an orthonormal wavelet transform. Bound.-Layer Meteor., 70, 307-326.

Hill, R. J., 1996: Corrections to Taylor's frozen turbulence approximation. Atmos. Res., 40, 153-175.

Hills, A. J., and P. R. Zimmerman, 1990: Isoprene measurement by ozone induced chemiluminescence. Anal. Chem., 62, 1055-1060.

Howell, J. F., and L. Mahrt, 1994: An adaptive multiresolution data filter: Applications to turbulence and climatic time series. $J$. Atmos. Sci., 51, 2165-2178. 
Irvine, M. R., and Y. Brunet, 1996: Wavelet analysis of coherent eddies in the vicinity of several vegetation canopies. Phys. Chem. Earth, 21, 161-165.

Kader, B. A., and A. M. Yaglom, 1991: Spectra and correlation functions of surface layer atmospheric turbulence in unstable thermal stratification. Turbulence and Coherent Structures, O. Metais and M. Lesieur, Eds., Kluwer Academic Press, 388-412.

Kaimal, J. C., and J. J. Finnigan, 1994: Atmospheric Boundary Layer Flows: Their Structure and Measurements. Oxford University Press, 289 pp.

Katul, G. G., 1994: A model for sensible heat flux probability density function for near-neutral and slightly stable atmospheric flows. Bound.-Layer Meteor., 71, 1-20.

—, and M. B. Parlange, 1994: On the active role of temperature in surface layer turbulence. J. Atmos. Sci., 51, 2181-2195.

- , and - 1995: The spatial structure of turbulence at production wavenumbers using orthonormal wavelets. Bound.-Layer Meteor., 77, 153-172.

— detached eddy motion in the atmospheric surface layer using Lorentz wavelet filtering. Bound.-Layer Meteor., 77, 153-172.

$\longrightarrow$, and - 1998: Identification of low-dimensional energy containing/flux transporting eddy motion in the atmospheric surface layer using wavelet thresholding methods. J. Atmos. Sci., 55, 377-389.

- M. B. Parlange, and C. R. Chu, 1994: Intermittency, local isotropy, and non-Gaussian statistics in atmospheric surface layer turbulence. Phys. Fluids, 6, 2480-2492.

—, J. D. Albertson, C. I. Hsieh, P. S. Conklin, J. T. Sigmon, M. B. Parlange, and K. K. Knoerr, 1996a: The inactive eddy motion and the large-scale turbulent pressure fluctuations in the dynamic sublayer. J. Atmos. Sci., 53, 2512-2524.

—, C. I. Hsieh, R. Oren, D. Ellsworth, and N. Phillips, 1996b: Latent and sensible heat flux predictions from a uniform pine forest using surface renewal and flux variance methods. Bound.Layer Meteor., 80, 249-282.

—, R. Oren, D. Ellsworth, C. I. Hsieh, and N. Phillips, 1997a: A Lagrangian dispersion model for predicting $\mathrm{CO}_{2}$ sources, sinks, and fluxes in a uniform loblolly pine stand. J. Geophys. Res., 102, 9309-9321.

—, C. I. Hsieh, G. Kuhn, D. Ellsworth, and D. Nie, 1997b: Turbulent eddy motion at the forest-atmosphere interface. J. Geophys. Res., 102, 13 409-13 421.

LaRue, J. C., and P. A. Libby, 1981: Thermal mixing layer downstream of half-heated turbulence grid. Phys. Fluids, 24, 597603 .

- _ - and D. V. R. Seshadri, 1981: Further results on the thermal mixing layer downstream of a turbulence grid. Phys. Fluids, 24, 1927-1933.

Leclerc, M. Y., K. C. Beissner, R. H. Shaw, G. den Hartog, and H. H. Neumann, 1990: The influence of atmospheric stability on the budgets of the Reynolds stress and turbulent kinetic energy within and above a deciduous forest. J. Appl. Meteor., 29, 916933.

Lu, C.-H., and D. R. Fitzjarrald, 1994: Seasonal and diurnal variations of coherent structures over a deciduous forest. Bound.-Layer Meteor., 69, 43-69.

Lumley, J. L., 1965: Interpretation of time spectra measured in high intensity shear flows. Phys. Fluids, 8, 1056-1062.

- 1986: Evolution of non-self-preserving thermal mixing layer. Phys. Fluids, 29, 3976-3981.

Ma, B. K., and Z. Warhaft, 1986: Some aspects of thermal mixing layer in grid turbulence. Phys. Fluids, 29, 3114-3120.

Mahrt, L., 1991: Eddy asymmetry in the sheared heated boundary layer. J. Atmos. Sci., 48, 472-492.

—, and J. Howell, 1994: The influence of coherent structures and microfronts on scaling laws using global and local transforms. J. Fluid Mech., 260, 247-270.
Meneveau, C., 1991: Analysis of turbulence in the orthonormal representation. J. Fluid Mech., 232, 469-520.

Moritz, E., 1989: Heat and momentum transport in an oak forest canopy. Bound.-Layer Meteor., 49, 317-329.

Mulhearn, P. J., and J. J. Finnigan, 1978: Turbulent flow over a very rough, random surface. Bound.-Layer Meteor., 15, 109-132.

Paw U, K. T., Y. Brunet, S. Collineau, R. H. Shaw, T. Maitani, J. Qiu, and L. Hipps, 1992: On coherent structures in turbulence above and within agricultural plant canopies. Agric. For. Meteor., 61, 55-68.

—, J. Qiu, H. B. Su, T. Watanabe, and Y. Brunet, 1995: Surface renewal analysis: A new method to obtain scalar fluxes. Agric. For. Meteor., 74, 119-137.

Qiu, J., K. T. Paw U, and R. H. Shaw, 1995: Pseudo-wavelet analysis of turbulent patterns in three vegetation layers. Bound.-Layer Meteor., 72, 177-204.

Raupach, M. R., 1979: Anomalies in flux-gradient relationships over forests. Bound.-Layer Meteor., 16, 467-486.

- 1988: Canopy transport processes. Flow and Transport in the Natural Environment: Advances and Applications, W. L. Steffem and O. T. Denmead, Eds., Springer-Verlag, 95-127.

_ , and A. S. Thom, 1981: Turbulence in and above canopies. Ann. Rev. Fluid Mech., 13, 97-129.

_ J. J. Finnigan, and Y. Brunet, 1989: Coherent eddies in vegetation canopies. Australian Conf. on Heat and Mass Transfer, Christchurch, New Zealand, 75-90.

- , R. A. Antonia, and S. Rajagopalan, 1991: Rough-wall turbulent boundary layers. Appl. Mech. Rev., 44, 1-25.

- J. J. Finnigan, and Y. Brunet, 1996: Coherent eddies and turbulence in vegetation canopies: The mixing layer analogy. Bound.-Layer Meteor., 78, 351-382.

Shaw, R. H., G. den Hartog, and H. H. Neumann, 1988: Influence of foliar density and thermal stability on profiles of Reynolds stress and turbulence intensity in a deciduous forest. Bound.Layer Meteor., 45, 391-409.

_ , Y. Brunet, J. J. Finnigan, and M. R. Raupach, 1995: A wind tunnel study of air flow in waving wheat: Two-point velocity statistics. Bound.-Layer Meteor., 76, 349-376.

Szilagyi, J., G. G. Katul, M. B. Parlange, J. D. Albertson, and A. T. Cahill, 1996: The local effect of intermittency on the inertial subrange energy spectrum of atmospheric surface layer. Bound.Layer Meteor., 79, 35-50.

Taylor, G. I., 1938: The spectrum of turbulence. Proc. Roy. Soc. London, 164A, 476-490.

Tennekes, H., and J. L. Lumley, 1972: A First Course in Turbulence. The MIT Press, 300 pp.

Thompson, N., 1979: Turbulence measurements above a pine forest. Bound.-Layer Meteor., 16, 293-310.

Townsend, A. A., 1961: Equilibrium layers and wall turbulence. $J$. Fluid Mech., 11, 97-120.

_, 1976: The Structure of Turbulent Shear Flow. Cambridge University Press, $428 \mathrm{pp}$.

Turner, B. J., and M. Y. Leclerc, 1994: Conditional sampling of coherent structures in atmospheric turbulence using the wavelet transform. J. Atmos. Oceanic Technol., 11, 205-209.

,-- M. Gauthier, K. E. Moore, and D. R. Fitzjarrald, 1994: Identification of turbulence structures above a forest canopy using a wavelet transform. J. Geophys. Res., 99, 1919-1926.

Vidakovic, B., 1995: Unbalancing data with wavelet transformations. Proc. Wavelet Applications in Signal and Image Processing III, San Diego, CA, SPIE, 845-857.

Wilson, J. D., 1989: Turbulent transport within the plant canopy. Estimation of Areal Evapotranspiration, T. A. Black, D. L. Spittlehouse, M. D. Novak, and D. T. Price, Eds., International Association of Hydrological Sciences, 43-80.

Wyngaard, J. C., and S. F. Clifford, 1977: Taylor's hypothesis and high frequency turbulence spectra. J. Atmos. Sci., 34, 922-929.

Yee, E., R. Chan, P. R. Kosteniuk, C. A. Biltoft, and J. F. Bowers, 1996: Multiscaling properties of concentration fluctuations in dispersing plumes revealed using an orthonormal wavelet decomposition. Bound.-Layer Meteor., 77, 173-207. 